\title{
Concepts of management of plastic surgery services during the coronavirus disease 2019 pandemic
}

\author{
Sammy Al-Benna ${ }^{1}$ (1)
}

Received: 16 May 2020 / Accepted: 2 July 2020 / Published online: 13 July 2020

(C) Springer-Verlag GmbH Germany, part of Springer Nature 2020

The recent emergence of a novel coronavirus, severe acute respiratory syndrome coronavirus 2 (SARS-CoV-2), and the disease it causes, coronavirus disease 2019 (COVID-19), has rapidly developed into a global pandemic and public health emergency [1-4]. As of 14 June 2020, a total of 7,895,800 accumulated cases and 432,882 deaths have been reported worldwide in 215 countries and regions, with an overall mortality rate of about $1 \%$ [5]. There will be variability in rates, peaks, and timing for each nation, and while at present one cannot precisely forecast many aspects, all plastic surgeons should prepare. In contrast to Europe, sub-Saharan Africa is on the upswing of the COVID-19 curve, and Cape Town is its current epicentre [5].

The current COVID-19 situation is challenging and presents a rapidly evolving set of circumstances which places a strain on our healthcare systems and providers. It also forces difficult choices about whether plastic surgical care can and should be delayed or reprioritized. The objectives of healthcare systems in response to the COVID-19 pandemic are to reduce morbidity and mortality, minimize disease transmission, protect healthcare personnel, and preserve healthcare system functioning.

Trauma is a mainstay of the need for plastic surgery expertise and will continue to be so the COVID-19 pandemic [5, 6]. All of us, even trainee, specialist, and retired plastic surgeons, must co-operate appropriately in the arena with the greater corps of healthcare professionals for the benefit of the public. This includes proper use and respect for the needs of supplies such as personal protective equipment and resources such as intensive care unit needs.

Sammy Al-Benna

albenna@sun.ac.za

1 Division of Plastic and Reconstructive Surgery, Faculty of Medicine and Health Sciences, Stellenbosch University and Tygerberg Academic Hospital, Francie van Zijl Drive, PO Box 241, Cape Town 8000, South Africa
Plastic surgery might not appear to be in the forefront of COVID-19 treatment; nevertheless, all physicians have a function to perform which must be prepared. Plastic surgeons and trainees may be required to function outside of their specialty after extra refresher training [2]. At present, about one quarter of them have taken on other respiratory and critical care roles in the hospital. Yet, the aspects of plastic surgery practice are no less critical than those of any other specialty.

In the reality of our health system, most of our preCOVID-19 theatre lists were "scheduled-urgent" cases, but now all non-emergency work has been restricted. However, the emergency patients, who are primarily linked to trauma (e.g. major burns, open fractures, extensive soft tissue trauma), continue to require admission and surgical care $[6,7]$. This requires preserving some form of plastic and reconstructive surgical capability in all scenarios.

Plastic surgeons' aim is to put patient's needs first. However, in the COVID-19 public health emergency, this role is shifting. Their responsibility to the common good may override the interests of an individual patient in some circumstances. Onset of COVID-19 during recovery from an operation complicates recovery and prolong the hospital stay, as well as expose healthcare professionals who would then have to be excluded from healthcare work [8]. While some cases can be delayed indefinitely, the majority of the plastic surgery cases performed are associated with progressive disease (e.g. skin cancer, soft tissue sarcoma, head, and neck cancer) that will continue to progress at variable, disease-specific rates. As these conditions persist and, in many cases, advance in the absence of surgical intervention, it is important to recognize that the decision to cancel or perform elective surgery should be made in the setting of many factors, both medical and logistical. Clear, open, and transparent decision-making is also particularly 
important during the pandemic response. Indeed, delaying some cases risks having them reappear as more severe emergencies at a time when they will be less easily handled.

Patients must be informed of local and national service constraints and be given the opportunity to understand their planned treatment within allowable resources [6, 7]. At present, decision-making may be within present ethical/practice standards and follow accepted guidelines and protocols. However, should the burden on health services continue to escalate as has been seen in other countries, decision-making may be more extraordinary. In these conditions, it is critical that when the decision is made, both the decision process and decision made is well documented.

The COVID-19 pandemic is putting healthcare systems under mounting pressure. COVID-19 is a dangerous disease that demands the participation of everyone to end this pandemic. All of us, trainee, specialist, and retired surgeons, must co-operate appropriately in the arena with the greater corps of healthcare professionals for the benefit of the public $[9,10]$ Surgical decision-making, always the most important aspect of care, has taken on an added layer of complexity in the face of the COVID-19 a pandemic when considering the ramifications of delaying the intervention at hand [9].

\section{Compliance with ethical standards}

Conflict of interest The author declares that he has no conflict of interest.
Ethical approval No ethical approval required.

Informed consent Not applicable.

\section{References}

1. Mayer HF, Persichetti P (2020) Plastic surgery during the COVID19 pandemic times. Eur J Plast Surg 43:361-362

2. Al-Benna S (2020) Impact of COVID-19 on surgical registrars' education and training. S Afr J Surg 58:10-13

3. Al-Benna S (2020) Availability of COVID-19 information from national and international aesthetic surgery society websites. Aesthet Plast Surg 44:1043-1046

4. Al-Benna S, Gohritz A (2020) Availability of COVID-19 information from national plastic surgery society websites. Ann Plast Surg. https://doi.org/10.1097/SAP.0000000000002447

5. Worldometer. COVID-19 Coronavirus pandemic. Available at: https://www.worldometers.info/coronavirus/ Date accessed: 14th June 2020

6. Al-Benna S (2013) Adequate specialised burn care services are essential at major trauma centres. Burns. 39:1495-1497

7. Al-Benna S (2013) Burn care facilities are lacking at major trauma centres in England. Burns. 39:533

8. Al-Benna S (2020) Management of hand surgery services during the coronavirus disease 2019 pandemic. J Hand Microsurg. https:// doi.org/10.1055/s-0040-1714440

9. Al-Benna S (2014) The paradigm of burn expertise: Scientia est lux lucis. Burns. 40:1235-1239

10. Al-Benna S, O’Boyle C (2014) Burn care experts and burn expertise. Burns. 40:200-203

Publisher's note Springer Nature remains neutral with regard to jurisdictional claims in published maps and institutional affiliations. 